\title{
TRANSPLANTE RENAL EM RECEPTOR COM SÍNDROME DE OCHOA E INSUFICIÊNCIA RENAL CRÔNICA - RELATO DE CASO
}

\section{Kidney transplantation in a patient with Ochoa's syndrome and chronic renal failure - a case report}

Diego Morais Gomes, Cláudia Maria Costa de Oliveira, Daniela Costa de Oliveira Santos, João Batista Gadelha Cerqueira, Márcia Uchoa Mota, Silvana Cristina Albuquerque Andrade, Leyla Castelo Branco Marques, Wilson Mendes Barroso, Regina Gomes Garcia, Ailson Gurgel Fernandes, Paula Frassinetti Castelo Branco Camurça Fernandes, João Batista Evangelista Jr

\section{RESUMO}

Os autores relatam o caso de uma paciente do sexo feminino, 21 anos, com diagnóstico de Síndrome de Ochoa e portadora de Insuficiência Renal Crônica (IRC) dialítica, submetida a transplante renal com doador falecido e sua evolução póstransplante. O Transplante renal é uma opção segura para pacientes com Síndrome de Ochoa que evoluem para IRC terminal, apesar do risco aumentado de infecção do trato urinário pós-transplante. A ampliação vesical com ou sem derivação urinária externa continente, além do cateterismo vesical intermitente deve ser recomendada para prevenção de dano ao enxerto renal.

Descritores: Bexiga Urinária Neurogênica; Insuficiência Renal, Transplante Renal.

Instituição:

Unidade de Transplante Renal do Hospital Universitário Walter Cantídeo - Universidade Federal do Ceará (UFC).

\section{Correspondência:}

Claudia Maria Costa de Oliveira

Nefrologia, Universidade Federal do Ceara - Brasil

Rua Professor Jacinto Botelho, 500 / 500, CEP 60.810-050, Fortaleza/CE

Tel.: (85) 3241-2036 Fax: (85) 3366-8611

E-mail: claudiadrl@gmail.com

Recebido em:10.06.2011

\section{INTRODUÇÃO}

A Síndrome de Ochoa (SO) ou Síndrome Urofacial foi descrita inicialmente por Bernardo Ochoa em 1960, sendo caracterizada como uma uropatia obstrutiva funcional com dilatação do trato urinário superior (sintomatologia de bexiga neurogênica, mas sem anormalidade neurogênica ou obstrutiva) ${ }^{1}$ e fácies invertida característica que aparenta tristeza, dor ou desconforto e é evidenciada principalmente durante o sorriso. É uma rara desordem autossômica recessiva ligada ao cromossomo 10q23-q24 (gene HPSE2), ${ }^{2}$ existindo pouco mais de 100 casos relatados na literatura nos últimos 40 anos. $^{3-5}$ Pacientes com essa síndrome geralmente apresentam hidronefrose, hidroureter e distúrbios miccionais, resultantes de bexiga neurogênica, além de infecções urinárias de repetição, podendo evoluir com pielonefrite. A evolução para insuficiência renal é freqüente devido ao diagnóstico tardio e há poucos relatos de transplante $(\mathrm{Tx})$ renal bem sucedido nesses pacientes.

\section{OBJETIVO}

Relatar o caso de uma paciente com SO submetida a Tx renal e sua evolução pós-Tx renal. 


\section{RELATO DE CASO}

ACOS, sexo feminino, 21 anos, parda, estudante, submetida a Tx renal com doador falecido em novembro/2008. O tempo em hemodiálise antes do transplante foi de sete anos.

Antecedentes Patológicos: A doença renal primária foi diagnosticada aos 12 anos de idade (maio/99) como bexiga neurogênica espástica e uropatia obstrutiva funcional bilateral, já apresentando disfunção renal no momento do diagnóstico. Ao exame físico inicial, foram detectados dedos extranuméricos no pé esquerdo e fácies típica de Síndrome de Ochoa (Figura 1).

Figura 1 - Fácies típica da Síndrome de Ochoa evidenciada com o sorriso

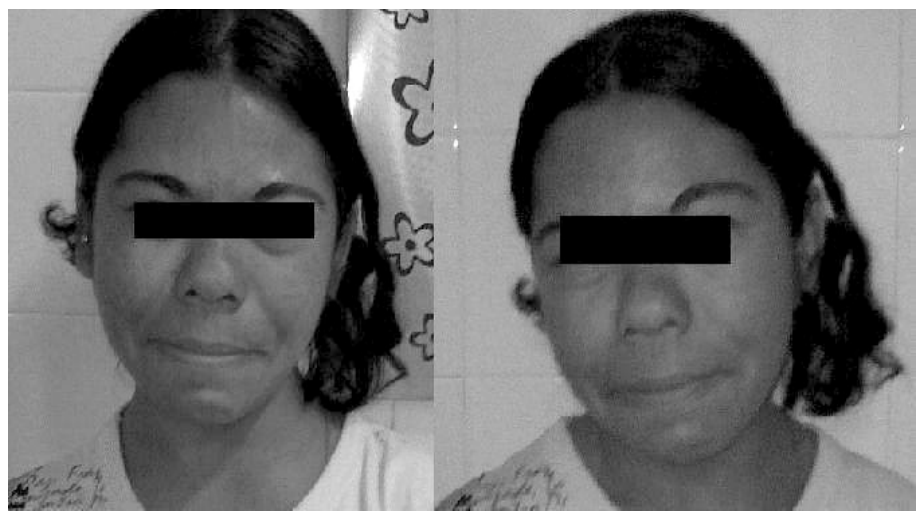

A avaliação urológica inicial (maio/1999) compreendeu ultrassonografia renal, uretrocistografia Miccional (UCM) e urodinâmica. O ultrassom revelava hidronefrose e hidroureter bilateral. A UCM mostrava refluxo vesico-ureteral (RVU) bilateral grave com bexiga de esforço e na urodinâmica foi evidenciada capacidade vesical $80 \mathrm{ml}$, bexiga não complacente, pressão intravesical $140 \mathrm{~cm} \mathrm{H} 2 \mathrm{O}$, pressão de perda elevada $(140 \mathrm{~cm} \mathrm{H} 2 \mathrm{O})$, pressão uretral de $40 \mathrm{~cm} \mathrm{H} 2 \mathrm{O}$ e ausência de hiperatividade do detrusor.

A paciente ficou em uso de oxibutinina e foi submetida a pielostomia bilateral em junho/99, complicando com infecções do trato urinário (ITUs) de repetição sintomáticas, tendo sido internada várias vezes com febre, dor epigástrica e descarga purulenta uretral. Evoluiu posteriormente para insuficiência renal crônica (IRC) terminal, tendo iniciado terapia dialítica aos 14 anos (2001).

Avaliação pré-transplante renal: No preparo para o Tx renal, foram realizadas ampliação vesical e derivação urinária externa continente do tipo Mitrofanoff (apendicovesicostomia) em julho/2003 e, posteriormente, nefrectomia bilateral, tendo sido liberada para Tx renal por equipe urológica em setembro/2005, com indicação de cateterismo vesical intermitente pós-Tx renal através do orifício da região umbilical. UCM pré-Tx (julho/ 2004) mostrou bexiga ampliada com contornos irregulares e divertículos em sua parede, sem RVU e com resíduo pós-miccional importante; estudo urodinâmico evidenciou capacidade vesical diminuída (150 $\mathrm{ml})$, bexiga não complacente $(3 \mathrm{ml} / \mathrm{cm} \mathrm{H} 2 \mathrm{O})$, pressão do detrusor na capacidade cistométrica máxima (CCM) elevada (48 cm H2O), pressão de perda de zero cm H2O, com hiperatividade do detrusor e sem perda urinária. Uma segunda UCM em julho/2005 demonstrou bexiga cirúrgica de contornos irregulares, com boa repleção, capacidade vesical de $130 \mathrm{ml}$, resíduo vesical pós-esvaziamento de pequena quantidade e sem RVU durante esforço miccional.

Evolução do transplante renal: A paciente foi submetida a Tx renal com doador falecido em 2008. O doador era do sexo feminino, 28 anos, causa mortis AVC hemorrágico pós-eclâmpsia e creatinina no óbito de 1,5 mg/dl. O tempo de isquemia fria foi de 27 horas e a compatibilidade HLA de 5 mismatches, com painel de anticorpos reativos de $0 \%$. A anastomose urinária foi uretero-ureteral e a paciente ficou com cateter ureteral duplo J durante os primeiros 60 dias pós-transplante. Foi utilizado como terapia imunossupressora inicial esquema tríplice com tacrolimus (Tac), micofenolato mofetil e prednisona, além de terapia de indução com basiliximab. A paciente evoluiu com função retardada do enxerto, sendo necessária hemodiálise nos primeiros 22 dias. A biópsia renal realizada aos 20 dias pós-Tx revelou alterações borderline (primeira biópsia renal aos oito dias pós-Tx com material inadequado). Foi então iniciada Timoglobulina (dose total: $7 \mathrm{mg} / \mathrm{Kg}$ ) e suspenso Tac, uma vez que a paciente continuava com disfunção do enxerto e em diálise, após o que evoluiu com boa diurese. Na reintrodução do Tac, observouse nova elevação de creatinina, tendo-se optado por conversão para sirolimus aos 33 dias pós-Tx, com boa resposta da função renal (creatinina atual de $1,0 \mathrm{mg} / \mathrm{dl}$ ). O tempo de internamento inicial foi de 35 dias. Após o Tx renal, manteve cateterismo vesical intermitente quatro a seis vezes por dia através de orifício de apendicovesicostomia na região umbilical (Figura 2).

Figura 2 - Cicatriz decorrente de cateterismo vesical em região umbilical

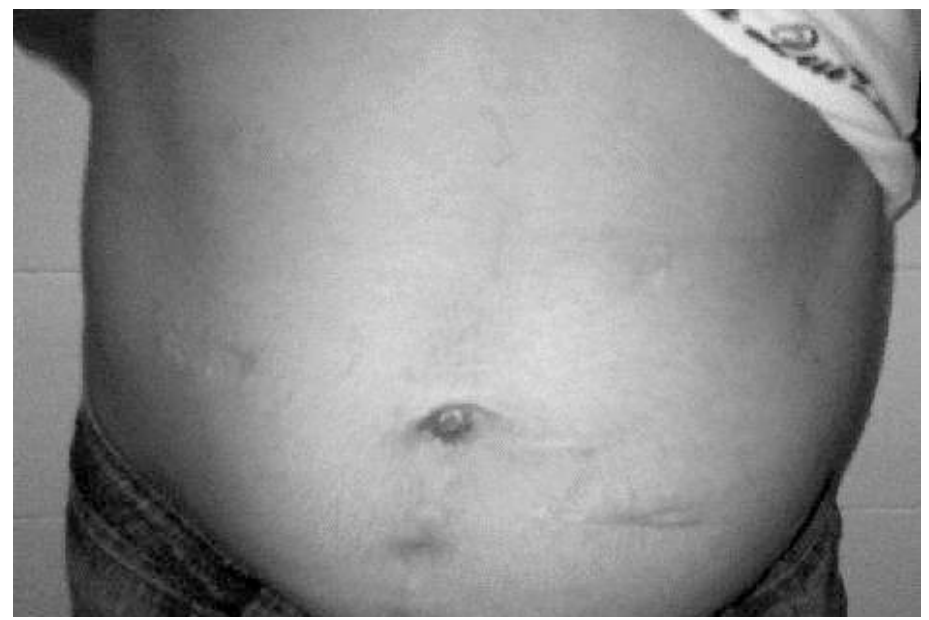

Em 32 meses de seguimento pós-Tx renal, apresentou oito episódios de ITU, sendo três episódios de ITU precoce ( $<6$ meses: aos 13 dias, 54 dias e 5 meses) e cinco episódios tardios (> 24 meses: 24, 25, 27, 28 e 30 meses pós-Tx). O agente etiológico foi Escherichia coli em três episódios, Klebsiella pneumoniae em quatro e Enterobacter cloacea em um episódio.

Apresentou-se com quadro de pielonefrite (dor no enxerto, febre e calafrios) em sete episódios e cistite em um episódio. Em um dos episódios de pielonefrite, a paciente complicou com sepse, disfunção renal aguda (creatinina máxima de 4,2 mgdl) e abscessos intra-renais, sendo necessário uso de meropenem por 28 dias.

A creatinina sérica aos 6,12 e 32 meses pós-tx renal foi de 1,0; 1,0 e $1,1 \mathrm{mg} / \mathrm{dl}$, respectivamente. Atualmente encontra-se em uso de sirolimus $1 \mathrm{mg} / \mathrm{dia}$, micofenolato mofetil $360 \mathrm{mg}$ duas vezes/dia e prednisona $5 \mathrm{mg} / \mathrm{dia}$.

\section{DISCUSSÃO}

O envolvimento simultâneo da bexiga e dos músculos faciais vem do fato de que a micção normal é um reflexo do tronco cerebral centrado na formação reticular e tem uma íntima relação anatômica com a origem dos nervos faciais. ${ }^{6} \mathrm{O}$ centro da micção localizado na formação reticular divide a mesma localização topográfica com 


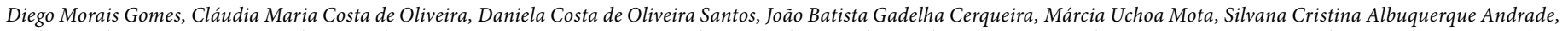

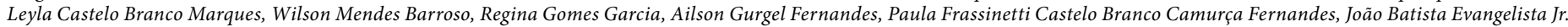

o centro do riso e do choro localizado acima do núcleo respiratório e facial. Lesões nessa área podem produzir dissinergia manifestada por disfunção da expressão facial e disfunção do esvaziamento de urina e fezes. A inversão da expressão facial que caracteriza a Síndrome de Ochoa não é um defeito estrutural facial, mas uma expressão disfuncional. Quando os pacientes sentem dor, choram ou estão tristes, a expressão facial é a mesma de indivíduos normais, mas não com o sorriso.

Estudos que relatam casos de Síndrome de Ochoa mostram igual prevalência entre sexos e maior freqüência em filhos de pais com estreito parentesco. Há relatos de que, aproximadamente $2 / 3$ desses pacientes, apresentam constipação de intensidade moderada a grave. $^{2} \mathrm{~A}$ presença de hidrocefalia, devido à estenose do aqueducto de Sylvius e atraso no desenvolvimento psicomotor também foram relatados em alguns pacientes. No presente caso, os pais não eram consaguíneos, de forma semelhante ao relatado por Yashwanth et al., ${ }^{7}$ embora a maioria dos relatos refira consaguinidade.

A Síndrome de Ochoa é um distúrbio genético raro, com freqüente evolução para IRC, mas há poucos relatos na literatura nacional e internacional da evolução pós-Tx renal de pacientes com essa síndrome.

Pacientes com anomalias do trato urinário inferior eram considerados candidatos de risco para realização de Tx renal. No entanto, a investigação do trato urinário inferior, através da urodinâmica pré-Tx e técnicas de reconstrução do trato urinário inferior com ampliação vesical e derivações urinárias ampliaram as indicações e a taxa de sucesso do transplante renal nesse grupo de pacientes. ${ }^{9}$

O presente estudo mostra um caso de ampliação vesical pré-Tx. A técnica utilizada foi ileocistoplastia continente utilizando-se o princípio de Mitrofanoff. A paciente evoluiu com disfunção precoce do enxerto, mas atualmente apresenta função renal normal (creatinina: 1,0-1,1mg/dl).

Um resultado semelhante foi demonstrado por Dawahra et al. ${ }^{10}$ em nove pacientes submetidos a Tx renal com derivação urinária continente pré-Tx. Neste estudo a técnica utilizada para a derivação urinária foi a bolsa de Koch em seis pacientes e a bolsa de Mainz em três pacientes. A sobrevida do enxerto foi de $77 \%$ com seguimento de 67 meses.

O tipo de derivação utilizada não parece ter importância na sobrevida do enxerto. Surange et al., ${ }^{11}$ utilizando o conduto ileal externo (Bricker) em 59 pacientes submetidos a Tx renal, mostrou resultados semelhantes aos estudos com derivação continente. ${ }^{10,12}$

O principal problema no pós-operatório é a dificuldade de esvaziamento do conduto, o que pode ocasionar infecção urinária com prejuízo da função do enxerto em longo prazo. No entanto, a literatura enfatiza a segurança da utilização do cateterismo intermitente nesse grupo de pacientes. $\mathrm{O}$ regime de cateterismo intermitente tem sido utilizado por mais de duas décadas em pacientes submetidos a transplante renal sem aumentar a taxa de perda do enxerto. ${ }^{13}$

O cateterismo vesical intermitente é uma ferramenta importante contra as ITUs recorrentes, mas a combinação de cateterismo intermitente e RVU persistente pode ser um gatilho para ITUs febris,${ }^{14}$ o que pode contribuir para a morbi-mortalidade do receptor do enxerto.

A prevalência de ITU em Tx renal de pacientes com SO não é conhecida. A ITU é a infecção bacteriana mais comum em receptores de transplante renal, apresentando incidência variável entre $6-86 \%$ (em média $30-35 \%$ dos pacientes). A maioria dos episódios ocorre no primeiro ano de Tx..$^{15}$ A ITU pós-Tx é mais fácil de detectar no pós-operatório precoce, e as infecções que ocorrem nos primeiros três meses associam-se com pielonefrite em $90 \%$ dos casos. No presente caso, a paciente apresentou oito episódios de ITU precoces e cinco tardios, e o episodio que complicou com septicemia ocorreu tardiamente aos 24 meses pós-Tx.

Uma análise da base americana de dados renais (United States Renal Data System - USRDS) revela que ITU tardia (após seis meses de Tx) associa-se com pobre sobrevida do enxerto renal e maior mortalidade. ${ }^{16}$

A ITU pós-Tx pode aumentar a mortalidade do receptor, sendo o trato urinário a fonte de septicemia em 40-60\% dos casos de sepse. ${ }^{17}$ Por outro lado, $7 \%$ das ITU complicam com septicemia. A ITU pós-Tx pode manifestar-se como bacteriúria assintomática, cistite aguda, pielonefrite aguda ou septicemia, ${ }^{18}$ sendo que no caso relatado a clínica foi de cistite em apenas um episódio e pielonefrite em sete episódios ( $87 \%$ dos casos), sendo que em um deles a manifestação foi mais grave, com sepse associada.

Além disso, as bactérias podem estimular a secreção de TNFalfa, INF-gama, IL-1, IL-6 e IL-8, que são citocinas envolvidas no processo de rejeição, podendo desta forma aumentar o risco de rejeição aguda/ crônica após uma ITU. Muller et al. encontraram que após três anos de Tx, pacientes com número maior de ITU tinham um risco significativamente aumentado de rejeição crônica. ${ }^{19}$

A ITU pode também estar associada à reativação do citomegalovírus. Não é incomum observar replicação ativa de CMV, duas a três semanas após episódio de ITU febril, mesmo que ocorra anos pósTx. A ITU leva à produção de TNF-alfa, que é um fator chave na reativação do CMV. ${ }^{20}$ Portanto, espera-se que em pacientes com ITU de repetição haja menor sobrevida do enxerto, além de uma qualidade de vida inferior aos pacientes sem esse problema.

No caso de nossa paciente, em três anos de transplante foram necessárias quatro internações para terapia de ITU, totalizando 82 dias de internamento, o que pode ter tido um impacto na qualidade de vida.

Apesar da necessidade do procedimento de cateterismo vesical intermitente e do risco aumentado de ITU pós-Tx, a evolução da paciente mostra que o Tx renal é uma opção terapêutica adequada para pacientes com esta disfunção vesical.

Este relato de caso confirma os dados encontrados na literatura que demonstram os bons resultados do transplante renal em pacientes com disfunção do trato urinário inferior que tenham sido submetidos previamente a algum procedimento de derivação urinária.

Embora o transplante renal resulte em uma melhor qualidade de vida e sobrevida para os pacientes com Sindrome urofacial em relação aos pacientes que permanecem em tratamento dialítico, outras anormalidades associadas à síndrome como a constipação, a encoprese (descritas em 60\% e 33\% dos casos, respectivamente) 14 e a disfunção cognitiva, podem continuar a ser um problema limitante da qualidade de vida nesses pacientes. ${ }^{21}$

\section{CONCLUSÃO}

Embora o diagnóstico de Síndrome de Ochoa seja infrequente, o mesmo deve ser investigado em pacientes com disfunção miccional do tipo bexiga neurogênica espática, sendo seu reconhecimento de essencial importância para que se possa fazer um diagnóstico precoce e adotar uma terapêutica adequada, prevenindo assim a deterioração progressiva e irreversível da função renal. O Tx renal é uma opção segura para pacientes com SO que evoluem para IRC terminal, apesar do risco aumentado de ITU pós-Tx. A ampliação vesical com ou sem derivação urinária externa continente, além do cateterismo vesical intermitente deve ser recomendada para prevenção de dano ao enxerto renal. 


\section{ABSTRACT:}

Purpose: The authors report the case of a 21 years old female patient diagnosed with Ochoa's Syndrome and chronic renal failure (CRF), who had a deceased donor kidney transplant and her post-transplantation evolution. Kidney transplant is a safe treatment for renal patients with Ochoa's Syndrome that evolve to Chronic Renal Failure, despite the increased risk of UTI after transplantation. The vesical magnification with or without external continent urinary diversion added to the vesicle intermittent catheterization should be recommended to prevent damage to the renal graft.

Keywords: Urinary Bladder, Neurogenic; Renal Insufficiency; Kidney Transplantation

\section{REFERÊNCIAS:}

1. Ochoa B. Can a congenital dysfunctional bladder be diagnosed from a smile? The Ochoa syndrome updated. Pediatric Nephrology. 2004;19(1):6-12.

2. Ochoa B, Gorlin RJ. Urofacial (ochoa) syndrome. Am J Med Genet. 1987;27(3):661-7.

3. Nicanor FA, Cook A, Pippi-Salle JL. Early Diagnosis of the Urofacial Syndrome is Essential to Prevent Irreversible Renal Failure. International Braz. J. Urol. 2005;31(5):477-81.

4. Skálová S, Rejtar I, Novák I, Jüttnerová V. The Urofacial (Ochoa) Syndrome: First Case in the Central European Population. Prague Medical Report 2006;107(1):125-9.

5. Garcia-Minaur S, Oliver F, Yanez JM, Soriano JR, Quinn F, Reardon W1. Three new European cases of urofacial (Ochoa) syndrome. Clin Dysmorphol. 2001;10:165-70.

6. Ochoa B. The urofacial (Ochoa) syndrome revisited. J Urol. 1992;148:580-3.

7. Yashwanth R, Chandra N, Mohandas P, Gopinath PM. Urofacial (Ochoa) Syndrome - A Case Report. Int J Hum Genet. 2010;10(1-3):165-8.

8. Muñoz Fernández ME, Rodó Salas J, Grande Moreillo C, Morales Fochs L. Urofacial Ochoa's syndrome: a clinical case. Actas Urol Esp. 2001;25(8):578-

9. Parada B, Figueredo A, Mota A, Furtado A. Renal transplantation in patientswith lower urinary tract dysfunction. Transplant Proc. 2003;35(3):1089-90.

10.Dawahra M, Martin X, Tajra LC, Cloix P, Marechal JM, Garnier JL et al. Renal transplantation using continent urinary diversion: long-term followup. Transplant Proc. 1997;29(1-2):159-60.

11.Surange RS, Johnson RW, Tavakoli A. Kidney transplantation into a ileal conduit: a single center experience of 59 cases. J Urol.2003;170(5):1727-30.
12.Nahas WC, Mazzuchi E, Arap MA. Augmentation cystoplasty in renal transplantation: a good and safe option- experience with 25 cases. Urology 2002;60(5):770-4.

13. Shneidman RJ, Pulliam JP, Barry JM. Clean, intermittent self-catheterization in renal transplant recipients. Transplantation 1984;38:312-4.

14.Aydogdu O, Burgu B, Demirel F, Soygur T, Ozcakar ZB, Yalcmkaya F et al. Ochoa syndrome: a spectrum of urofacial syndrome. Eur J Pediatr. 2010;169:431-5.

15.Ricea JC, Safdarb N e The AST Infectious Diseases Community of Practice. Urinary Tract Infections in Solid Organ Transplant Recipients. Am J Transpl. 2009;9(Suppl 4):S267-72.

16.Abbott KC, Swanson SJ, Richter ER , Bohen EM, Agodoa LY, Peters TG et al. Late urinary tract infection after renal transplantation in the United States. Am J Kidney Dis. 2004;44(2):353-2.

17.Abbott KC, Oliver JD 3rd, Hypolite I, Lepler LL, Kirk AD, Ko CW et al. Hospitalizations for Bacterial Septicemia after Renal Transplantation in the United States. Am J Nephrol.2001;21:120-7.

18.Chuang P, Parikh CR, Langone A. Urinary tract infections after renal transplantation: a retrospective review at two US transplant centers. Clin Transplant. 2005,19(2):230-5.

19.Muller V, Becker G, Delfs M, Albrecht KH, Philipp T, Heemann U. Do urinary tract infections trigger chronic kidney transplant rejection in man? $\mathrm{J}$ Urol. 1998;159(6):1826-9.

20.de Souza RM, Olsburgh J. Urinary tract infection in the renal transplant patient. Nat Clin Pract Nephrol. 2008 ;4 (5):252-64.

21.Feng WC, Churchill BM. Dysfunctional elimination syndrome in children without obvious spinal cord diseases. Pediatr Clin North Am. 2001;48:1489504. 